\title{
Synthesis of a novel $p$-hydroxycinnamic amide with anticancer capability and its interaction with human serum albumin
}

\author{
YUAN-HONG JIAO $^{1}$, FA-YAN MENG ${ }^{2}$, GUI-BING ZHU ${ }^{1}$, LING-ZI RAN ${ }^{1}$, YU-FENG JIANG ${ }^{3}$ and QIAN ZHANG $^{1}$ \\ ${ }^{1}$ College of Chemistry and Chemical Engineering, Hubei Polytechnic University, Huangshi, Hubei 435003; \\ ${ }^{2}$ Pharmaceutical College, Guangxi Medical University, Nanning, Guangxi 530021; ${ }^{3}$ College of Materials \\ Science and Engineering, Hubei Polytechnic University, Huangshi, Hubei 435003, P.R. China
}

Received January 4, 2018; Accepted July 6, 2018

DOI: $10.3892 /$ etm.2018.7060

\begin{abstract}
In the present study, a novel $p$-hydroxycinnamic amide (E)-3-(4-hydroxy phenyl)-N-(4-(N-(5-meth oxypyrimidin-2-yl)-sulfamoyl)phenyl)acrylamide (HMSP) was synthesized and confirmed. In vitro cytotoxic assays indicated that HMSP was able to inhibit the proliferation of various cancer cell lines. The interaction between HMSP and human serum albumin (HSA) was examined by fluorescence, UV-Vis and circular dichroism (CD) spectra, in addition to molecular simulation. The fluorescence and UV-Vis spectra data indicated that the binding of HMSP with HSA was a static process. According to the fluorescence quenching calculation, the corresponding thermodynamic parameters, bimolecular quenching rate constant and apparent quenching constants were calculated. Van der Walls forces and hydrogen bonds were vital in the binding of HMSP on HSA. The distances between HSA and its derivatives were obtained. Furthermore, competitive experiments and molecular modeling results suggested that the binding of the compound on HSA mainly occurred in site I (sub-domain IIA). Changes in HSA conformation were observed from synchronous fluorescence and CD spectra, which were further investigated by molecular dynamic simulations.
\end{abstract}

\section{Introduction}

$p$-Hydroxycinnamic acid is a type of aromatic acid found in medicinal plants, exhibiting anticancer, antioxidant, antivirus, immunomodulatory, antiallergic, and other forms

Correspondence to: Mrs. Fa-Yan Meng, Pharmaceutical College, Guangxi Medical University, 22 Shuangyong Road, Nanning, Guangxi 530021, P.R. China

E-mail: mfy1114@yahoo.com.cn

Dr Qian Zhang, College of Chemistry and Chemical Engineering, Hubei Polytechnic University, 16 North Guilin Road, Huangshi, Hubei 435003, P.R. China

E-mail: zhangqian@hbpu.edu.cn

Key words: p-hydroxycinnamic amides, human serum albumin, synthesis, interaction, thermodynamics of pharmacological activity (1). Owing to these numerous biological activities, $p$-hydroxycinnamic acid is a useful medicine and is widely used in the chemical industry and in organic synthesis to develop a variety of aromatic amides with important activities (2). Substantial evidence exists to show that $p$-hydroxycinnamic derivatives are promising and leading compounds for the development of chemopreventive/anti-inflammatory agents $(3,4)$. $p$-hydroxycinnamic amides more effectively inhibit the growth of cancer cells than $p$-hydroxycinnamic acid (5). Furthermore, the $p$-hydroxycinnamic amides possess higher antioxidant activity against lipoperoxidation, thus increasing their capability to reduce carcinogenesis (6). One category of $p$-hydroxycinnamic derivatives takes advantage of sulfonamides as an amine for drug synthesis (7). Sulfonamides are a family of synthetic drugs used for the treatment of infections, and the amide moiety may be involved by changing the metabolic activity of the invading pathogenic microorganisms.

Previous studies have focused only on the bioactivities of $p$-hydroxycinnamic and its derivatives, with less attention focused on its ability to target its effects in human tissues. It is well established that the interaction between biomacromolecules and drugs can determine their distribution, effective concentration and metabolism in plasma, in addition to drug stability and safety during chemotherapeutic processes (8). In pharmacology, interactions between drugs and proteins can affect the bioactivities and toxicity of a drug (9). Therefore, the interaction of $p$-hydroxycinnamic derivatives with protein require consideration, as such an interaction is associated with drug transport, biological activity and clearance (10).

Serum albumin (SA) is the most abundant protein within the blood, and it considered to have a leading role in drug combination, processing and delivery (11). A previous study have investigated the interactions of various active molecules with SA or other serum components, which serve as carriers for these molecules in the blood (12). Following entry to the body, the drugs depend on SA to reach the lesion site, and then fulfil their therapeutic effect. The binding and dissociating constant of drugs and SA determines their in vivo activities. Human SA (HSA) is usually selected as a target protein molecule due to its pharmacological significance, stability, binding and transport properties.

In the present study, a novel $p$-hydroxycinnamic acid derivative, (E)-3-(4-hydroxyphenyl)-N-(4-(N-(5-meth 
oxypyrimidin-2-yl)-sulfamoyl)phenyl)acrylamide (HMSP) was synthesized and characterized, using sulfonamide drugs as an amine compound. Toxic experiments showed that HMSP inhibited cancer cell proliferation at micromolar concentrations. The thermodynamics of the interaction between HMSP and HSA were examined using fluorescence, UV-Vis absorbance spectroscopy, and molecular modeling methods. The binding site of HMSP was designated to site I, namely, the warfarin site on the HSA molecule, and the van der Waals interaction was found to be vital for the binding of HMSP to HSA. However, several hydrogen bonds existed. Synchronous fluorescence and circular dichroism (CD) spectra indicated that the binding of HMSP onto HSA altered the conformation of HSA, and this was further supported by molecular dynamic (MD) simulation results. The present study provided accurate and complete data to clarify the binding mechanisms of HMSP with HSA, which may prove valuable for understanding the effects of $p$-hydroxycinnamic acid derivative on serum function during the transportation process, and provide insight into its toxicity in vivo.

\section{Materials and methods}

Materials. HSA was obtained from Shanghai Yuanju Biological Technology Co., Ltd. (Shanghai, China) and stored under refrigeration at $4.0^{\circ} \mathrm{C}$. The $p$-hydroxycinnamic acid (chemical reagent grade) and sulfamethoxydiazine (chemical reagent grade) were purchased from Shenzhen Yuan Cheng Science and Technology Co., Ltd. (Shenzhen, China). A p-hydroxycinnamic acid derivative was synthesized through a condensation reaction of $p$-hydroxycinnamic acid with sulfamethoxydiazine. All other reagents were commercial products of analytical grade, and $\mathrm{ddH}_{2} \mathrm{O}$ was used for all solution preparations.

Apparatus and measurements. The X-ray diffraction data for the compound was recorded on a Bruker SMART CCD diffractometer and solved using the SHELXTL-97 program (13). The measurement of fluorescence spectra was completed on a RF-5301PC model spectrofluorometer (Shimadzu Corporation, Kyoto, Japan) equipped with a water bath $\left(\mathrm{T} \pm 0.1^{\circ} \mathrm{C}\right)$. The UV-Vis spectrum was recorded on a U-2000 spectrophotometer (Pharmacia; GE Healthcare Life Sciences, Uppsala, Sweden). The NMR spectra for compounds were collected on a Bruker $300 \mathrm{MHz}$. The MS spectra were collected on a Varian Saturn 2200 GCMS spectrometer. The IR spectra were analyzed using a Fourier transform infrared spectrophotometer (Spectrum 100; PerkinElmer, Inc., Waltham, MA, USA).

General procedure for the synthesis of HMSP. The amide compounds of HMSP were prepared from the condensation and deacetylation reaction of $p$-acetyl cinnamoyl chloride and sulfonamide. The $p$-acetyl cinnamoyl chloride was obtained as reported previously (8). At room temperature, condensation was completed in tetrahydrofuran, and concentrated hydrochloric acid $(0.5 \mathrm{ml})$ was used to remove the acetyl group at $60^{\circ} \mathrm{C}$, resulting in the corresponding amide compound.

HMSP is a white powder, yield $70 \%$, mp: $229-232^{\circ} \mathrm{C}$, MS: $467.2 \mathrm{~m} / \mathrm{z}$, calcd 468.11, ${ }^{1} \mathrm{H}-\mathrm{NMR}$ (300 MHz, DMSO- $d 6$, $\left.\delta \mathrm{ppm}\right)$ :

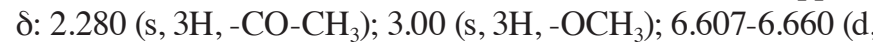
$1 \mathrm{H},-\mathrm{C}=\mathrm{C}-\mathrm{H}, J=15.9 \mathrm{~Hz})$; 7.201-7.229 (d, 2H, Ar-H, $J=8.4 \mathrm{~Hz})$; 7.622-7.675 (d, 1H, -C=C-H, J=15.9 Hz); 7.675-7.703 (d, 2H,
Ar-H, J=8.4 Hz); 7.853-7.984 (m, 4H, Ar-H); 8.300 (s, 2H, Py-H); 10.604 (s, 1H, -CO-NH); 11.431 (s, 1H, - $\mathrm{SO}_{2}-\mathrm{NH}$ ). Anal. calcd. for $\mathrm{C}_{22} \mathrm{H}_{20} \mathrm{~N}_{4} \mathrm{O}_{6} \mathrm{~S}$ : C, 56.40; H, 4.30; N, 11.96. Found: C, 56.50; H, 4.33; N, 11.89. IR data (KBr pellets, $\left.\mathrm{cm}^{-1}\right): 3,361(\mathrm{~m})$, 3,104 (w), 3,029 (w), 1,681 (m), 1,590 (s), 1,511 (s), 1,287 (s) and $1,092(\mathrm{~m})$.

Cytotoxicity assay. Human cervical carcinoma cell lines (HeLa), gastric cancer cell lines (SGC-7901), and pulmonary adenocarcinoma cell lines (A549) were maintained in DMEM supplemented (Gibco; Thermo Fisher Scientific, Inc., Waltham, MA, USA) with $10 \%$ heat-inactive fetal bovine serum (Gibco; Thermo Fisher Scientific, Inc.) at $37^{\circ} \mathrm{C}$. The cells were seeded into 96-well culture plates at $10^{4}$ cells per well and adhered overnight. Different concentrations (0, 10, 20, 40, 60, 80, 100, 150 and $200 \mu \mathrm{M})$ of HMSP were added to the cells. The cells were further incubated for $48 \mathrm{~h}$, following which the media was discarded, and 3-(4,5-dimethylthiazol)-2,5-diphenyltetrazolium bromide (MTT) solution $(0.5 \mathrm{mg} / \mathrm{ml})$ was added to each well. After $4 \mathrm{~h}$, the supernatant was discarded and $150 \mu \mathrm{l}$ DMSO was added into each well. Following incubation at $40^{\circ} \mathrm{C}$ and shaking for $10 \mathrm{~min}$, the absorbance of the plates was recorded at a wavelength of $570 \mathrm{~nm}$.

Spectroscopic experiments. The fluorescence emission spectra of HSA in each case was recorded with a 1-cm quartz cell using 15/4 nm slit widths. The emission spectra of HSA with or without HMSP were recorded at $310-450 \mathrm{~nm}$. A trace syringe was used to titrate HSA solutions $(1 \mu \mathrm{M})$ with HMSP. The final concentration of HMSP was in the range of $0-2 \mu \mathrm{M}$.

Synchronous fluorescence spectra of the HSA-HMSP systems were monitored under the same conditions as the emission spectra. The spectra of the HSA-HMSP systems were scanned in the wavelength ranges of 260-340 $\mathrm{nm}$ and 260-370 nm, and the differences between the excitation and emission wavelengths were 15 and $60 \mathrm{~nm}$, respectively.

The CD spectra of the HSA-HMSP systems were measured at room temperature under constant nitrogen flush $(260-200 \mathrm{~nm})$. The concentration of HSA was $1 \mu \mathrm{M}$ and the molar ratio of HSA to HMSP was set to 1:0.6, 1:1, 1:2 and 1:5. The $\alpha$-helix, $\beta$-sheet and unordered percentages were calculated using SELCON3 software (14).

Site marker competitive experiments. The titration fluorescence spectra of the HSA-HMSP system in the presence of the two typical site markers (warfarin and ibuprofen) were monitored using the same methods as described above. The concentrations of warfarin or ibuprofen were $1 \mu \mathrm{M}$. Increasing quantities of HMSP were then added into the HSA-warfarin or HSA-ibuprofen mixtures.

Molecular modeling. Molecular docking experiments were performed within the Surflex-Dock program implicated in the ssybyl 8.1 software package (15). The high-resolution crystal structures of HSA were retrieved from the RSCB Protein Data Bank (code: 1h9z) (16). The molecular structure of HMSP was constructed and minimized using the Tripos force field with Gasteiger-Marsili charges. The results of the best-fit binding conformations were analyzed and mapped using Visual Molecular Dynamics 1.9.1 software (17). 
Table I. $\mathrm{IC}_{50}$ values $(\mu \mathrm{M})$ of HMSP towards cancer cell lines.

\begin{tabular}{lc}
\hline Cell & $\mathrm{IC}_{50}$ value \\
\hline HeLa & 22.2 \\
SGC7901 & 17.5 \\
A549 & 37.8 \\
\hline
\end{tabular}

HMSP, $p$-hydroxycinnamic acid derivative.<smiles>COc1cnc(NS(=O)(=O)c2ccc(NC(=O)/C=C/c3ccc(O)cc3)cc2)nc1</smiles>

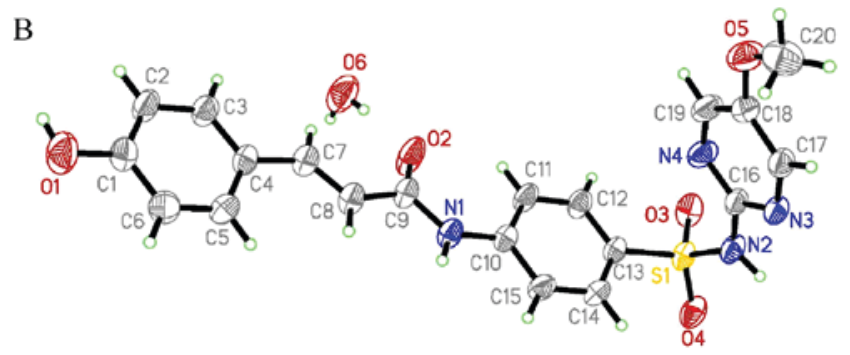

Figure 1. (A) Structure of HMSP. (B) ORTEP view showing the atom-labeling scheme with thermal ellipsoids drawn at $30 \%$ probability for HMSP. HMSP, $p$-hydroxycinnamic acid derivative.

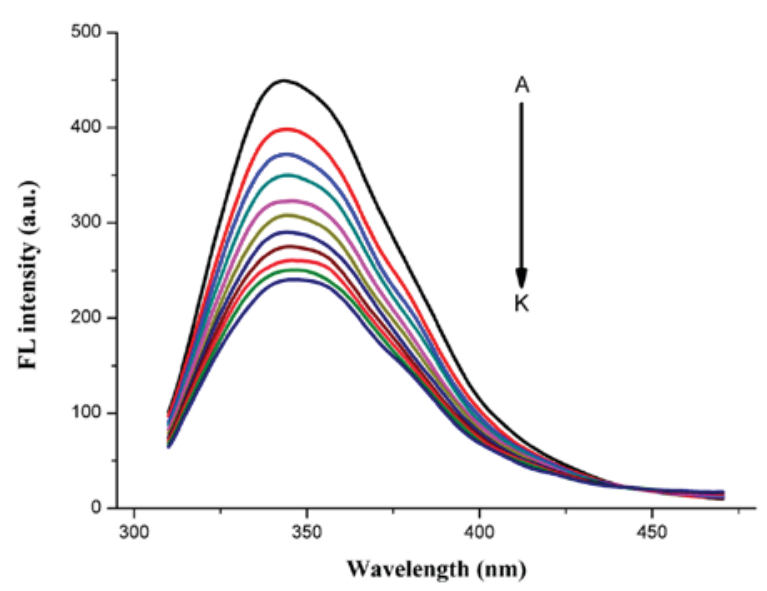

Figure 2. Fluorescence emission spectra of HSA in the presence of different concentrations of HMSP at $298 \mathrm{~K}$. From A to K, concentrations of HMSP were $0-2 \mu \mathrm{mol} / 1$ at an interval of $0.2 \mu \mathrm{mol} / 1$. c(HSA) $=1 \mu \mathrm{mol} / 1$. HSA, human serum albumin; HMSP, $p$-hydroxycinnamic acid derivative; FL, fluorescence.

The MD simulation was performed in the present study using Amber 14 software and Amber ff99SB force field (18). The MD simulations were started from the best-fit docking conformation. A periodic cubic TIP3P water box was used for the HSA-HMSP complex. The electroneutrality of the solvated system was then maintained by adding sodium ions. Prior to the MD simulations, the HSA-HMSP system was submitted to a two-step energy minimization procedure. First, a 2,000-step minimization of the solvent and ions only was performed, followed by a 2,000-step minimization of the entire system. The HSA-HMSP complex was gradually heated in an isothermal-isochoric (NVT) ensemble to $300 \mathrm{~K}$ with a period of 200 ps. The systems were then submitted to a 200 ps MD simulation in an isothermal-isobaric (NPT) ensemble at 1 atm and $300 \mathrm{~K}$. The protein backbone was harmonically constrained with a weak restraint $\left(10 \mathrm{kcal} / \mathrm{mol} \AA^{2}\right)$ in the minimization, heating, and equilibrating phases. Finally, $50 \mathrm{~ns}$ production MD simulation was performed. A cutoff of $10 \AA$ was set for short-range interactions, whereas long-range electrostatic interactions beyond the cutoff were handled with the particle mesh Ewald method. Covalent bonds involving hydrogen atoms were constrained by the SHAKE algorithm. The time step was set to $2 \mathrm{fs}$, and the trajectory was recorded every 2 ps.

\section{Results and Discussion}

Characterization of HMSP. HMSP was characterized by X-ray diffraction measurements. A perspective view of these two compounds in the atomic labeling system is shown in Fig. 1. The crystal in the space group $P 2(1) / c$ had the following unit cell parameters: $a=8.188$ (5) $\AA, b=13.656$ (8) $\AA, c=18.821$ (11) $\AA, \beta=96.781(7)^{\circ}, V=2090$ (2) $\AA^{3}, R=0.0468, w R_{2}=0.1425$ and $Z=4$. The X-ray data have been deposited at the Cambridge Crystallographic Data Centre (CCDC no. 838365).

Cytotoxicity assay. The cytotoxicity of HMSP was investigated using a typical MTT method. Three cancer cell lines, human cervical carcinoma cell lines (HeLa), gastric cancer cell lines (SGC-7901), and pulmonary adenocarcinoma cell lines (A549) were selected for investigation. The cytotoxic results of HMSP towards three different cancer cell lines are shown in Table I. It was observed that HMSP efficiently inhibited the proliferation of each of the cancer cell lines at micromolar concentration. In addition, as HMSP was synthesized by modifying $p$-hydroxycinnamic acid, its cellular toxicity was compared with its precursor. It was observed that HMSP exhibited improved anticancer capability, compared with $p$-hydroxycinnamic acid. These results demonstrated that HMSP may be used as a potential anticancer agent.

Fluorescence quenching and quenching mechanism. The binding of a drug on serum proteins brings about increased drug solubility in blood, reduced toxicity, and protection against oxidation of the bound drug (19). The present study investigated the binding parameters of HMSP to HSA. Proteins possess intrinsic fluorescence due to the existence of tryptophan, tyrosine and phenylalanine. The involvement of tyrosine and tryptophan residues in drug-HSA interactions can be evaluated by different excitation wavelengths. At an excitation wavelength of $280 \mathrm{~nm}$, the tryptophan and tyrosine residues are excited, whereas a wavelength of $295 \mathrm{~nm}$ excites tryptophan residues (20). As shown in Fig. 2, HMSP quenched HSA fluorescence excited at $295 \mathrm{~nm}$. The intrinsic fluorescence of HSA was gradually quenched compared with that of pure HSA solution, which indicated that the compounds were able to interact with HSA to form a compound-HSA complex.

It is known that there are two types of quenching mechanisms of protein fluorescence, namely static quenching and dynamic quenching (21). In static quenching, the quencher binds to Phe, Tyr, or Trp residues of HSA, and a nonfluorescent 
Table II. Quenching constants for the interaction of $p$-hydroxycinnamic acid with human serum albumin at different temperatures and $\mathrm{pH}$ 7.4.

\begin{tabular}{lcccr}
\hline Temperature $(\mathrm{K})$ & $K_{\mathrm{SV}} /\left(\mathrm{x} 10^{5} 1 \cdot \mathrm{mol}^{-1}\right)$ & $k_{\mathrm{q}} /\left(\mathrm{x} 10^{13} 1 \cdot \mathrm{mol}^{-1} \mathrm{~S}^{-1}\right)$ & $R^{2}$ & $\mathrm{SD}$ \\
\hline 298 & 4.27 & 4.27 & 0.9972 & 0.07 \\
304 & 3.48 & 3.48 & 0.9997 & 0.02 \\
310 & 2.98 & 2.98 & 0.9930 & 0.08 \\
\hline
\end{tabular}

$R^{2}$, correlation coefficient; $K_{\mathrm{SV}}$, Stern-Volmer quenching constant; $k_{\mathrm{q}}$, quenching rate constant of HSA; SD, standard deviation for $K_{\mathrm{SV}}$.

fluorophore-quencher complex is formed, resulting in decreased fluorescence intensity of HSA. Dynamic quenching represents the diffusion of the quencher towards the fluorophore in the lifetime of the excited state and, in the process of contact, the fluorophore returns to the ground state without photon emission (22). The quenching mechanism between compounds and HSA can be investigated by the Stern-Volmer equation (23):

$$
F_{0} / F=1+k_{\mathrm{q}} \tau_{0}[Q]=1+K_{\mathrm{SV}}[Q]
$$

where $F_{0}$ and $F$ are the fluorescence intensities of HSA with or without HMSP; $K_{\mathrm{SV}}$ is the Stern-Volmer quenching constant; $k_{\mathrm{q}}$ represents the quenching rate constant of HSA; $\tau_{0}$ is the average life-time of HSA without the compound $\left(\tau_{0}=10^{-8} \mathrm{~s}\right)(19)$; and $[Q]$ is the concentration of compound. In a dynamic quenching process, the quenching rate constant of quenchers with the biopolymer is $<2.0 \times 10^{10} 1 \cdot \mathrm{mol}^{-1} \cdot \mathrm{s}^{-1}$. If the $K_{\mathrm{q}}$ is substantially higher than this value, it can be inferred that the quenching process is not caused by dynamic quenching, but may be due to the static quenching as a result of the complex formation.

The Stern-Volmer plots of $F_{0} / F$ for HSA, vs. $[Q]$ at the concentrations of $0-2.0 \times 10^{-6} \mathrm{~mol} \cdot \mathrm{l}^{-1}$ based on equation (1) at 298 , 304 and $310 \mathrm{~K}$ are shown in Fig. 3. The comparatively good linear associations $\left(R^{2}=0.9971,0.9997\right.$ and 0.9930$)$ indicate that a single quenching mechanism, whether dynamic or static quenching, occurred at $37^{\circ} \mathrm{C}$. The values of $K_{\mathrm{SV}}$ and $k_{\mathrm{q}}\left(=K_{\mathrm{Sv}} / \tau_{0}\right)$ obtained from the plots are listed in Table II. The values of $k_{\mathrm{q}}$ were all $>2.0 \times 10^{10} 1 \cdot \mathrm{mol}^{-1} \cdot \mathrm{s}^{-1}$, which suggested that the quenching mechanism arose mainly from the formation of the HSA-HMSP complex and was controlled by a static mechanism. The results also suggested that the binding between HMSP and HSA was strong, and that HMSP was stored and carried by HSA in the plasma.

The dynamic and static quenching processes can be differentiated by their disparate dependence on temperature. Higher temperatures lead to a high rate of diffusion, resulting in increased dynamic quenching. By contrast, higher temperatures usually cause the decomposition of the weakly-bound complex, resulting in less static quenching (24). To clarify the quenching mechanism, fluorescence data at three different temperatures were analyzed using the Stern-Volmer equation. As shown in Fig. 3, the $K_{\mathrm{SV}}$ was negatively correlated with temperature, suggesting that the possible quenching mechanism of the HMSP-HSA binding process was caused by complex formation, rather than by dynamic collision.

To further determine the static quenching mechanism, the UV-Vis absorption spectra of HSA, HMSP and HSA-HMSP

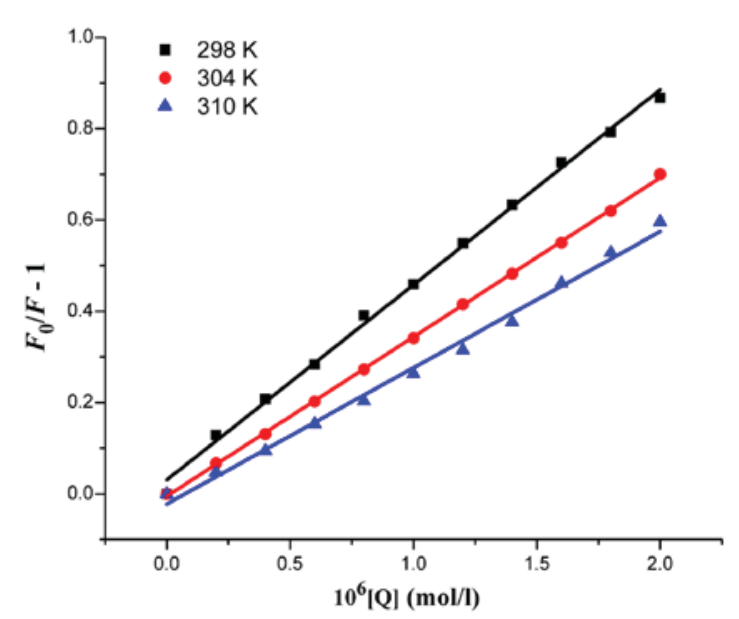

Figure 3. Stern-Volmer plots for the $p$-hydroxycinnamic acid derivative (HMSP)/human serum albumin system at different temperatures.

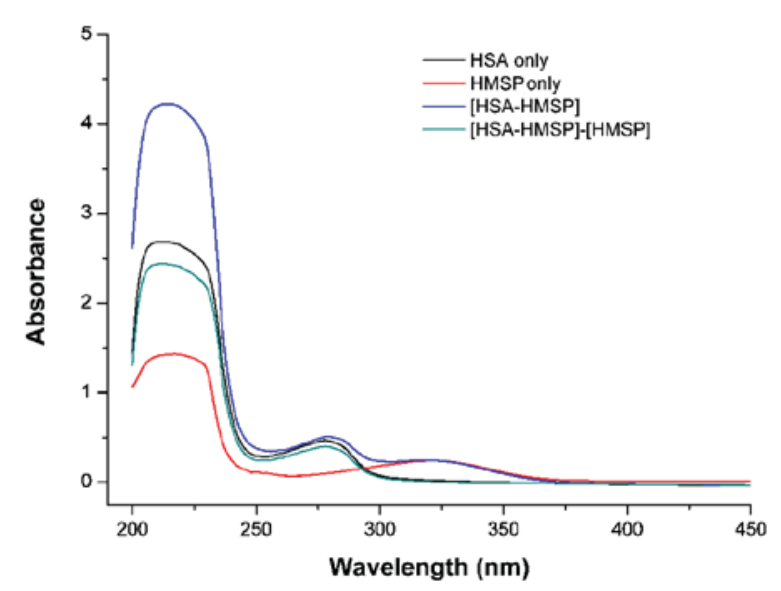

Figure 4. UV-Visible absorption spectra of HSA in the presence of HMSP. $\mathrm{c}(\mathrm{HSA})=\mathrm{c}(\mathrm{HMSP})=1 \mu \mathrm{mo} / 1$. HSA, human serum albumin; HMSP, $p$-hydroxycinnamic acid derivative.

systems were measured. It is known that the formation of a protein-compound complex causes a change of the absorbance spectra. By contrast, a collisional quenching process only alters the excited states of the fluorophores, and does not alter the absorption spectra (25). As shown in Fig. 4, the difference in absorption spectra between HSA-HMSP and HMSP were not superimposed with the absorption spectra of HSA. Therefore, the fluorescence quenching was caused by the formation of complex HSA-compounds, and this indicated primarily a static quenching mechanism. 
Table III. Thermodynamic parameters of the $p$-hydroxycinnamic acid-human serum albumin system.

\begin{tabular}{lccccc}
\hline Temperature $(\mathrm{K})$ & $K_{\mathrm{a}}\left(\mathrm{x} 10^{4} \mathrm{l} \cdot \mathrm{mol}^{-1}\right)$ & $R^{\mathrm{a}}$ & $\Delta H^{\theta}\left(\mathrm{kJ} \cdot \mathrm{mol}^{-1}\right)$ & $\Delta G^{\theta}\left(\mathrm{kJ} \cdot \mathrm{mol}^{-1}\right)$ & $\Delta S^{\theta}\left(\mathrm{J} \cdot \mathrm{mol}^{-1} \cdot \mathrm{K}^{-1}\right)$ \\
\hline 298 & 4.801 & 0.983 & -31.01 & -35.29 & 14.38 \\
304 & 3.696 & 0.999 & & -35.38 & 0.9993 \\
310 & 1.983 & 0.999 & & -35.46 & \\
\hline
\end{tabular}

${ }^{\mathrm{a} C}$ Correlation coefficient for $K_{\mathrm{a}}$ values; ${ }^{\mathrm{b}}$ correlation coefficient for van't Hoff plot. $K_{\mathrm{a}}$, binding constant of the protein and quencher; $\Delta H$, enthalpy change; $\Delta G$, free energy change; $\Delta S$, entropy change.
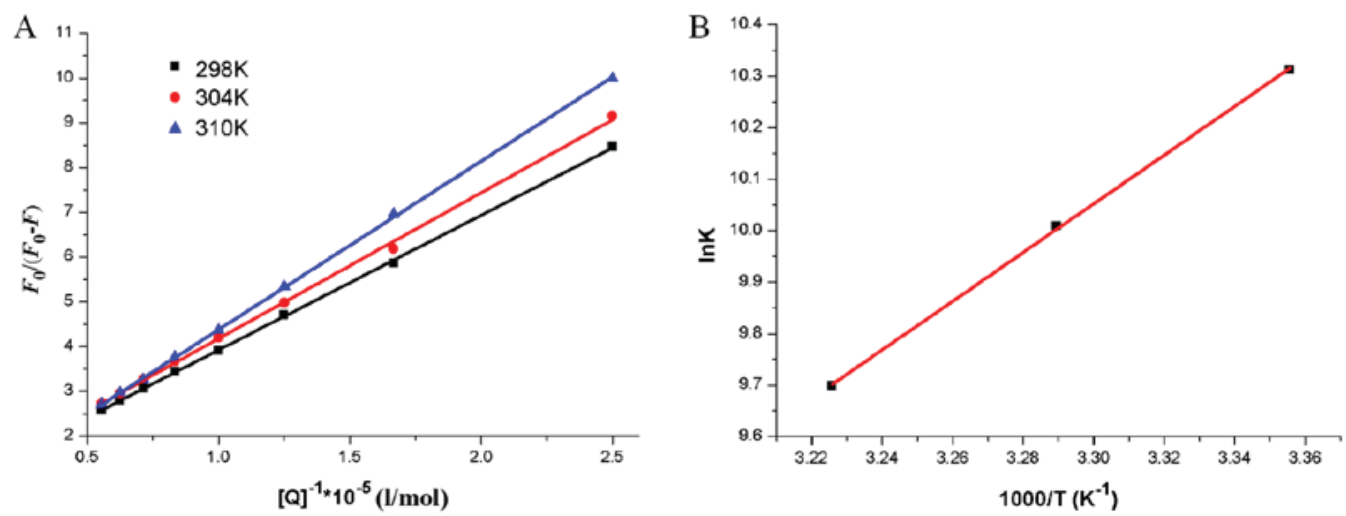

Figure 5. (A) Modified Stern-Volmer plots for the HMSP/HSA system at different temperatures. (B) Van't Hoff plots of the HMSP/HSA system. HSA, human serum albumin; HMSP, $p$-hydroxycinnamic acid derivative.

Determination of binding modes. For the static quenching interaction, the quenching data were analyzed according to a modified Stern-Volmer equation (26):

$$
F_{0} / \Delta F=1 / f_{\mathrm{a}} K_{\mathrm{a}}[Q]+1 / f_{\mathrm{a}}
$$

where $K_{a}$ is the binding constant of the protein and quencher; and $f_{a}$ is the fraction of the initial fluorescence that can be quenched. The thermodynamic parameters can be calculated by the van't Hoff equation:

$$
\ln K_{a}=-(\Delta H / R T)+(\Delta S / R)
$$

where $K_{a}$ is the binding constant, $R$ is the gas constant and $\Delta \mathrm{S}$ is the entropy change. The enthalpy change $(\Delta H)$ can be estimated from the plot of $\ln K_{a}$, vs. $1 / T$. The free energy change $(\Delta G)$ can be calculated from the following equation:

$$
\Delta G=\Delta H-T \Delta \mathrm{S}=-R T \ln K_{a}
$$

Modified Stern-Volmer plots for the quenching of HSA by HMSP at different temperatures are shown in Fig. 5A and plots of the van't Hoff equation are shown in Fig. 5B. The binding parameters and the thermodynamic parameters of binding of HMSP to HSA are shown in Table III. The negative values for $\Delta G$ indicate that the binding process was spontaneous.

There are four types of non-covalent forces that contribute to the binding of ligands to biomacromolecules, including hydrogen bonds, van der Waals forces, electrostatic forces, and hydrophobic interactions. The details of these forces can be determined through thermodynamic experiments. Leckband and Subramanian (27) suggested the values and magnitude of thermodynamic parameters associated with various interactions. The negative $\Delta H$ and $\Delta S$ in the present study indicated that the main forces for the HMSP-HSA complex were van der Waals forces and hydrogen bonds.

Identification of binding site and binding distance. The crystal structure of HSA consists of three domains sequentially named I-III, each including two sub-domains (A and B). Several studies have shown that the regions of ligands bound to HSA are mainly located in hydrophobic cavities in sub-domains IIA and IIIA. These have been labeled site I and site II (28). To identify the compound binding sites on HSA, site marker competitive experiments were performed by utilizing warfarin and ibuprofen as site markers. The fluorescence spectra were recorded upon excitation at $295 \mathrm{~nm}$ and the Stern-Volmer quenching constant $\left(K_{s v}\right)$ was calculated according to equation (2). As shown in Fig. 6, the quenching constants of the system with warfarin showed a marked decrease for HMSP, whereas the constants obtained from the system with ibuprofen remained unchanged. This suggested that the binding of compounds to HSA were affected by adding warfarin, which indicated that the binding of HMSP to HSA was mainly located within site I (sub-domain IIA).

The spectral results indicated that HMSP can form complexes with HSA. For more information on the HMSP-HSA system, the distance $r$ between derivatives and HSA, according to Föster's non-radioactive energy transfer theory (29), were examined. Based on this theory, the efficiency $(E)$ of energy transfer between the donor and acceptor can be calculated as follows:

$$
E=1-\left(\mathrm{F} / \mathrm{F}_{0}\right)=\mathrm{R}_{0}^{6} /\left(\mathrm{R}_{0}{ }^{6}+\mathrm{r}^{6}\right)
$$

where $R_{0}$ is the critical distance when the transfer efficiency is $50 \% . \mathrm{R}_{0}{ }^{6}$ is calculated using the following equation: 
Table IV. Secondary structure of HSA with different concentrations of HMSP.

\begin{tabular}{lcccc}
\hline $\begin{array}{l}\text { Structural } \\
\text { components }\end{array}$ & $\alpha$-helix & $\beta$-sheet & $\beta$-turn & $\begin{array}{c}\text { Random } \\
\text { coil }\end{array}$ \\
\hline HSA & 0.583 & 0.064 & 0.138 & 0.222 \\
HMSP:HSA=0.5:1 & 0.572 & 0.065 & 0.139 & 0.224 \\
HMSP:HSA=1:1 & 0.561 & 0.069 & 0.146 & 0.240 \\
HMSP:HSA=2:1 & 0.535 & 0.069 & 0.153 & 0.246 \\
HMSP:HSA=5:1 & 0.518 & 0.072 & 0.159 & 0.251 \\
\hline
\end{tabular}

HSA, human serum albumin; HMSP, p-hydroxycinnamic acid derivative.

$$
\mathrm{R}_{0}{ }^{6}=8.8 \times 10^{-25} K^{2} N^{4} \Phi \mathrm{J}
$$

where $K^{2}$ is the spatial orientation factor between the emission dipole of the donor and the absorption dipole of the acceptor; $N$ is the refractive index of the medium; $\Phi$ is the fluorescence quantum yield of the donor; and $J$ is the overlap integral of the fluorescence emission spectrum of the donor and the absorption spectrum of the acceptor, determined according to the following equation:

$$
J=\left[\Sigma F(\lambda) \varepsilon(\lambda) \lambda^{4} \Delta \lambda\right] / \Sigma F(\lambda) \Delta \lambda
$$

where $F(\lambda)$ and $\varepsilon(\lambda)$ are the fluorescence intensity of HSA and molar absorption coefficient of HMSP at wavelength $\lambda$, respectively. $J$ can be evaluated by integrating spectra, as shown in Fig. 7. It has been reported for HSA that $K^{2}=2 / 3, \Phi=0.15$ and $N=1.36$ (22). According to equations (5-7), it was calculated that the value of $r$ between HMSP and HSA was $0.58 \mathrm{~nm}$, and this indicated that the probability of energy transfer from HSA to HMSP was high, and the quenching process was a non-radioactive transfer process.

Conformation investigation. Synchronous fluorescence spectra can provide information on the microenvironments close to the vicinity of the fluorophore molecules at a molecular level (30). The possible shift in position of maximum emission wavelength of biomolecules indicates changes of polarity around the chromospheres molecule. When the difference $(\Delta \lambda)$ between the wavelength of excitation and the emission is set to $15 \mathrm{~nm}$, the synchronous fluorescence spectrum gives the typical information of tyrosine. When $\Delta \lambda=60 \mathrm{~nm}$, the synchronous fluorescence presents the typical information of tryptophan (12). As shown in Fig. 8A and B, the synchronous fluorescence spectra for $\Delta \lambda=15 \mathrm{~nm}$ exhibited no marked shift at maximum emission peak, however, for $\Delta \lambda=60 \mathrm{~nm}$ the maximum emission wavelength exhibited a blue-shift, suggesting the binding of HMSP increased the hydrophobicity of the microenvironment around the Trp residue, whereas the microenvironment around the Tyr residue was not affected.

To further investigate the effects of HMSP on the conformation of HSA, CD experiments were performed. The CD spectrum is widely used to investigate the conformational changes of proteins or peptides in solution (31). As shown in

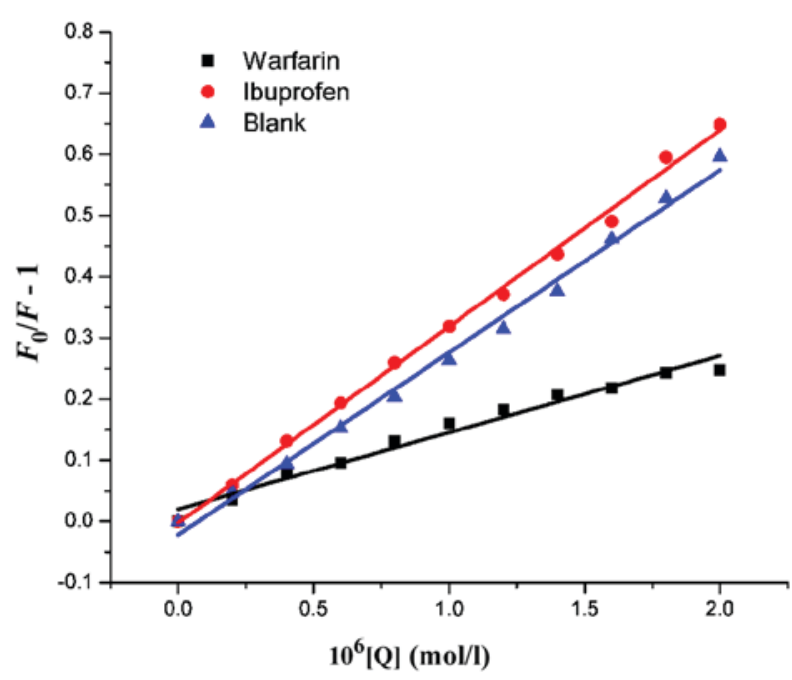

Figure 6. Stern-Volmer plots of the fluorescence quenching of HSA by the p-hydroxycinnamic acid derivative (HMSP) in the presence of site markers. $T=310 \mathrm{~K} \cdot \mathrm{c}($ warfarin $)=\mathrm{c}($ ibuprofen $)=\mathrm{c}(\mathrm{HSA})=1 \times 10^{-6} \mathrm{~mol} \cdot \mathrm{l}^{-1}$. HSA, human serum albumin.

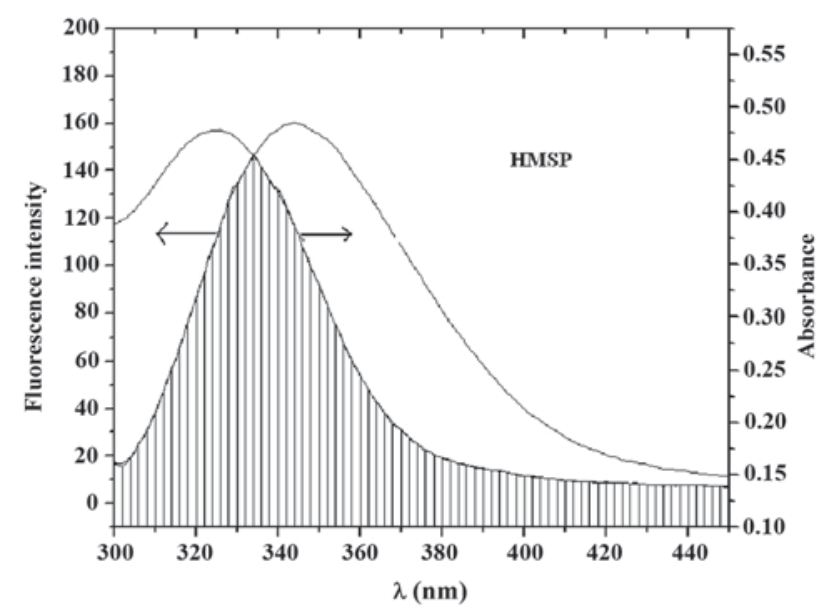

Figure 7. Overlapping of the fluorescence spectra with the absorption spectra of HMSP. HMSP, $p$-hydroxycinnamic acid derivative.

Fig. 9, HSA exhibited two typical negative peaks of 208 and $222 \mathrm{~nm}$, representing the $\alpha$-helix structure of HSA (32). HMSP was then added in increasing concentrations to measure the CD spectra of HSA. The contents of $\alpha$-helix, $\beta$-strand and other structures were calculated using SELCON3 software (Table IV). The free HSA in the absence of HMSP consisted of $\sim 58 \% \alpha$-helix, $\sim 20 \% \beta$-sheet and $\beta$-turn, and $\sim 22 \%$ random coils, and this was in agreement with a previous report (33). When the concentration of HMSP increased, the proportion of the secondary structural underwent marginal variations. At the molar ratio of 5:1, the $\alpha$-helix content decreased to $51.8 \%$ whereas he ratio of $\beta$-sheets and random coils increased to 23.1 and $25.1 \%$, respectively. These results indicated that the secondary structures of HSA were gradually disordered due to the formation of the HSA-HMSP complex.

Computational experiment. A docking experiment was used to investigate the binding modes between HMSP and HSA, particularly the binding sites and the binding conformations 

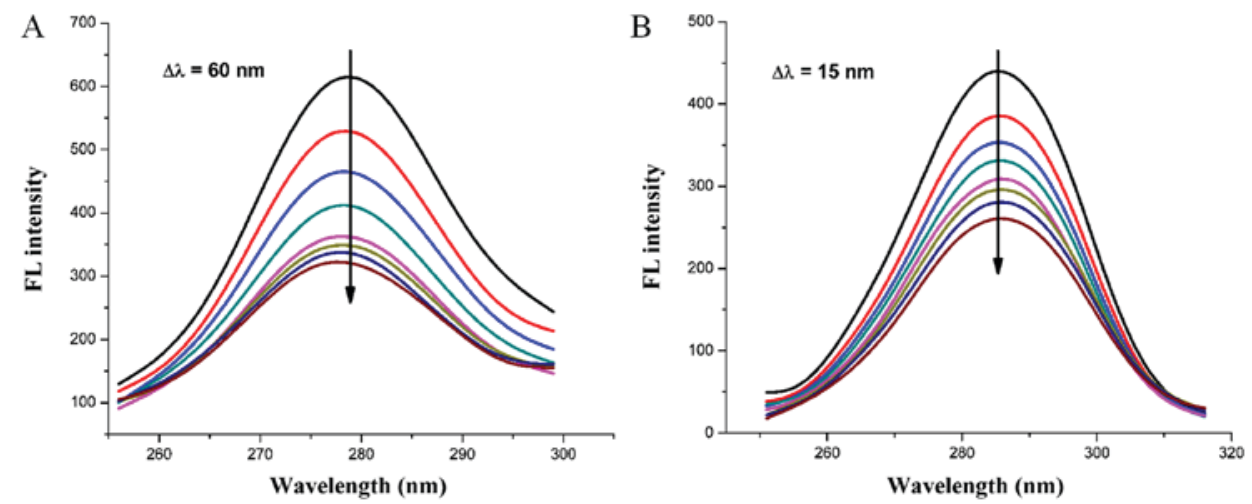

Figure 8. Synchronous fluorescence spectra of the interactions between human serum albumin and $p$-hydroxycinnamic acid derivative. (A) $\Delta \lambda=60 \mathrm{~nm}$; (B) $\Delta \lambda=15 \mathrm{~nm}$. FL, fluorescence. The arrows represent the maximum emission wavelength of human serum albumin.

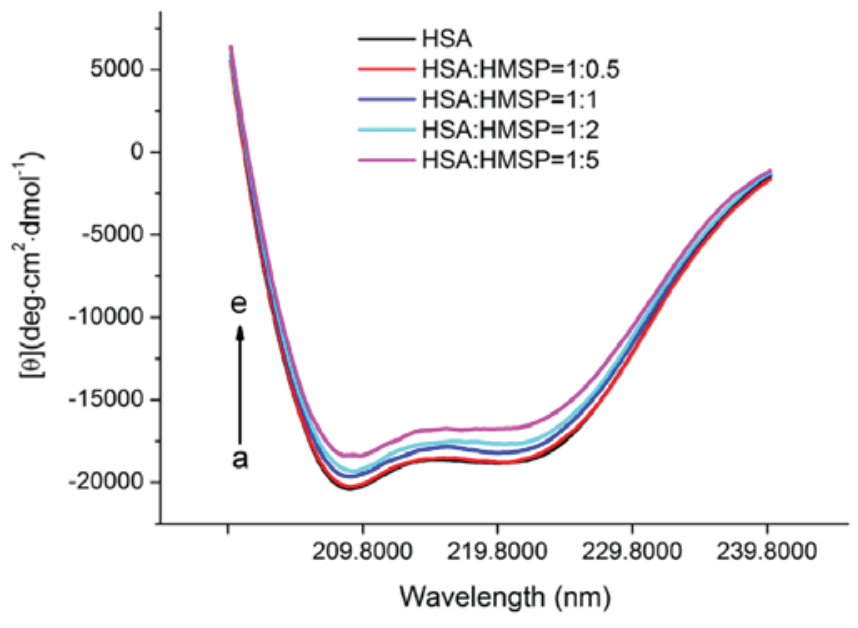

Figure 9. Circular dichroism spectra of the HSA-HMSP system. The arrow represents the maximum emission wavelength of HSA a) HSA; b) HSA:HMSP=1:0.5; c) HSA:HMSP $=1: 1$; d) HSA:HMSP $=1: 2$ e) HSA:HMSP=1:5. HSA, human serum albumin; HMSP, $p$-hydroxycinnamic acid derivative.

of the compounds. In the present study, the Surflex-Dock program (sybyl 8.1, tripos) was selected to identify the binding conformations of HMSP at the active HSA sites. In total, 20 conformations were obtained, and the conformation with the lowest free energy was selected for further analysis. The highest score-ranked result is shown in Fig. 10. The docking results indicated that HMSP bound in the hydrophobic pocket of sub-domain IIA (site I). The surrounding residues within $0.5 \mathrm{~nm}$ of HMSP were LEU198, LYS199, SER200, PHE211, TRP214, VAL215, ARG218, LEU219, ARG222, PHE223, LEU238, ILE290, and ALA291. As HMSP interacted mainly with hydrophobic side chains, it was inferred that the interaction between HMSP and HSA was mainly hydrophobic. This was consistent with the thermodynamic results. The phenyl and pyrimidine ring formed $\pi-\pi$ stacking with sidechains of PHE211 and TRP214. Furthermore, several hydrogen bonds existed between HMSP and sidechains of SER200 and a backbone of ILE290. The molecule docking results supported the indication that the binding of HMSP with HSA was stabilized by van der Waals and hydrogen bonds interactions.
MD simulation was performed on the HSA-HMSP complex to investigate the binding modes of HMSP and its effect on conformations of HSA. The convergence of the system equilibrium was examined by the time-dependent evolution of the root-mean-square deviation (RMSD) of backbone atoms to its initial structure. It was observed that the HSA was stable as the fluctuation of RMSD was small (Fig. 11A). The radius of gyration $(\mathrm{Rg})$ of free HSA and the HSA-HMSP complex were also calculated, as shown in Fig. 11B. The Rg values achieved equilibrium at $\sim 2.72$ and $2.85 \mathrm{~nm}$, respectively, suggesting that the $\mathrm{Rg}$ increased upon binding of HMSP to HSA. By comparing the final protein structure of the 50-ns MD simulation in the absence and presence of HMSP, it was found that the length of the cleft of HSA increased from 3.79 to $7.85 \mathrm{~nm}$ (Fig. 11C). This type of conformation change may have be caused by the insertion of HMSA in the center of the protein.

In conclusion, the interaction of a novel $p$-hydroxycinnamic amide HMSP with HSA was investigated under simulated physiological conditions by fluorescence and UV-Vis spectra, in addition to other molecular simulations. The binding constants, binding forces, binding distances and energy transfer parameters between HMSP and HSA were obtained. The results indicated that HMSP was able to bind to HSA molecules, the fluorescence quenching mechanism was static, and the binding reaction was spontaneous. The interactions were mainly enthalpy-driven, and hydrogen bonding, hydrophobic and van der Waals interactions were involved in the binding. The binding distance $(r)$ between HSA and HMSP was calculated as $0.58 \mathrm{~nm}$, indicating a high probability of energy transfer from HSA to HMSP. The binding site of HMSP on HSA was close to site I (sub-domain IIA). The synchronous fluorescence spectra indicated that HMSP affected the microenvironment of Tyr residues upon binding to HSA molecules. The CD spectrum and MD simulation results showed that the conformation of HSA became partially disordered upon binding of HMSP. The present study provided accurate and comprehensive data for identifying the binding mechanism of HMSP with HSA, and assists in understanding its effects on protein function during its transportation and distribution in plasma.

\section{Acknowledgements}

Not applicable. 
A

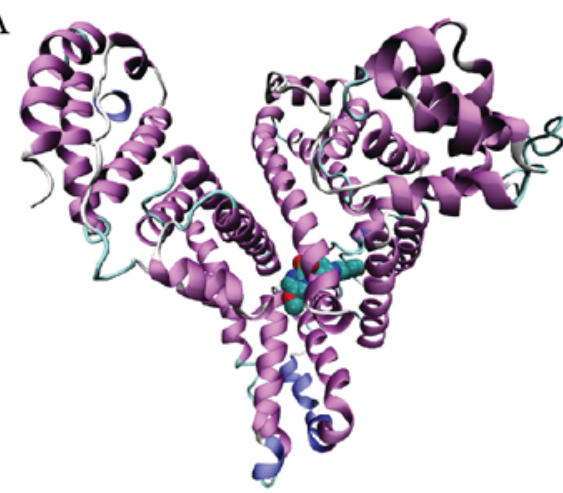

$\mathrm{B}$

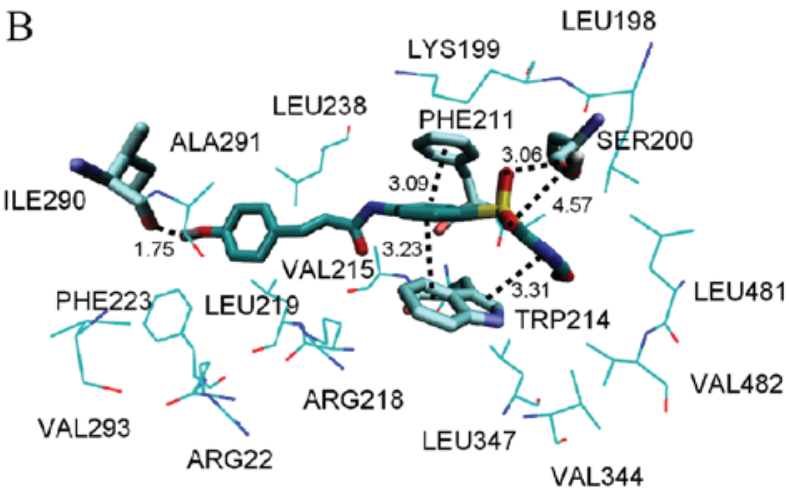

Figure 10. (A) Docking conformation of the HMSP/HSA complex. The image shows docked HMSP into the cavity of bovine serum protein; HMSP is colored by its atoms (carbon as blue, oxygen as red, nitrogen as green, and sulfur as yellow). (B) Interaction mode between HMSP and HSA, only residues $\sim 5 \AA$ of the ligand are shown. Nonpolar hydrogen atoms have been omitted for clarity. The distances are presented as $\AA$ values. HSA, human serum albumin; HMSP, $p$-hydroxycinnamic acid derivative.
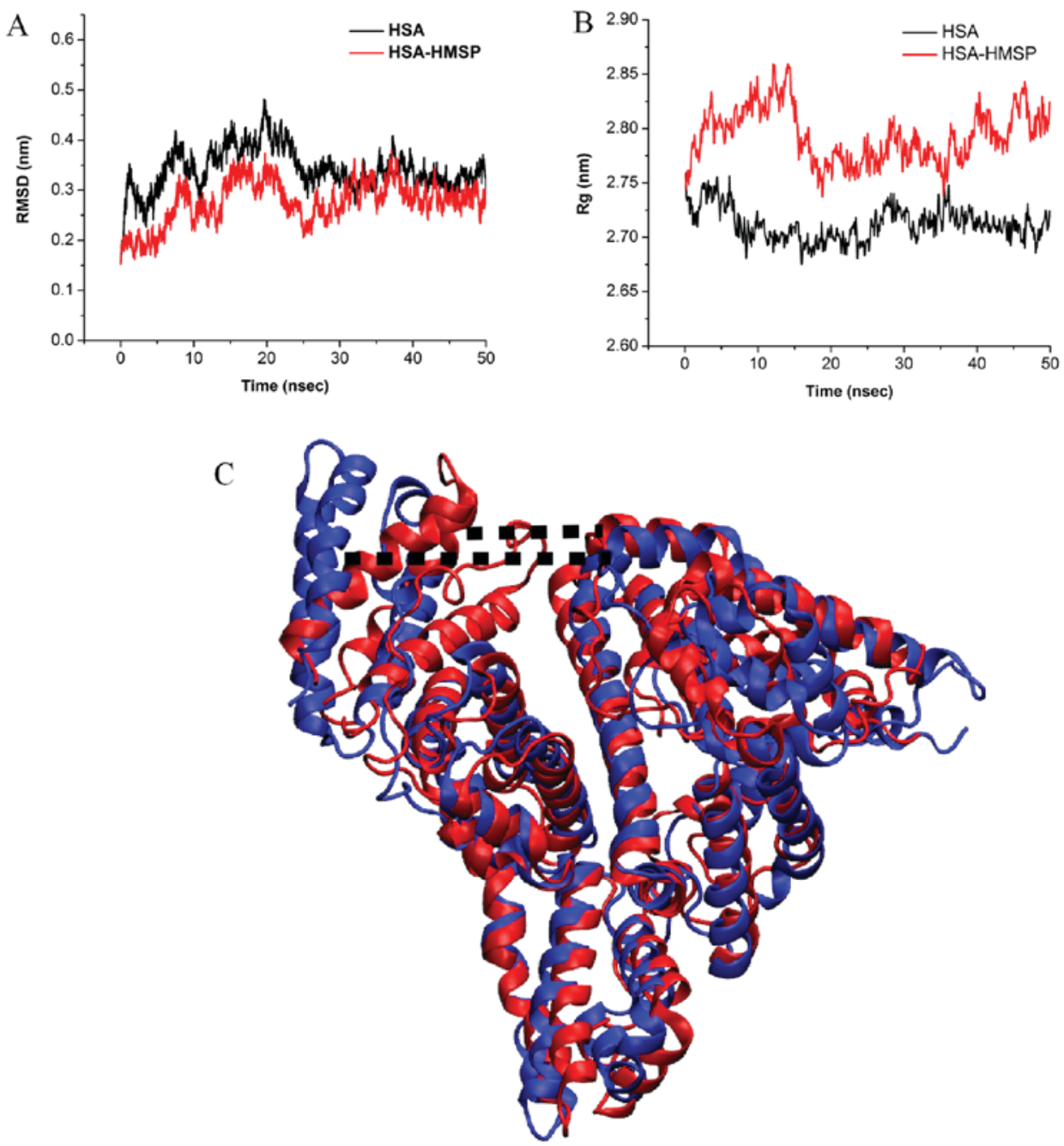

Figure 11. MD simulations. (A) Time dependence of RMSD values for HSA and the HSA-HMSP complex in 50 nsec MD simulations. (B) Time dependence of Rg values; (C) Superimposed final structure of protein from MD simulation of HSA (red) and HSA-HMSP (blue). MD, molecular dynamic; RMSD, root-mean-square deviation; Rg, radius of gyration; HSA, human serum albumin; HMSP, $p$-hydroxycinnamic acid derivative.

\section{Funding}

Thepresentstudy was financially supported bytheNatural Science Foundation of Guangxi (grant no. 2014GXNSFBA118053), the
College Student Innovation and Entrepreneurship Training Program of Hubei Province (grant no. 201710920034) and the Natural Science Foundation of Hubei Province of China (grant no. 2017CFB582). 


\section{Availability of data and materials}

The datasets used and/or analyzed during the current study are available from the corresponding author on reasonable request.

\section{Authors' contributions}

YHJ and FYM conceived the research. GBZ, LZR and YFJ conducted the experiments. QZ analyzed the data. YHJ and FYM wrote the manuscript. All authors read and approved the final manuscript.

\section{Ethics approval and consent to participate}

Not applicable.

\section{Patient consent for publication}

Not applicable.

\section{Competing interests}

The authors declare that they have no competing interests.

\section{References}

1. Calabriso N, Scoditti E, Massaro M, Pellegrino M, Storelli C, Ingrosso I, Giovinazzo $\mathrm{G}$ and Carluccio MA: Multiple anti-inflammatory and anti-atherosclerotic properties of red wine polyphenolic extracts: Differential role of hydroxycinnamic acids, flavonols and stilbenes on endothelial inflammatory gene expression. Eur J Nutr 55: 477-489, 2016.

2. Maury DK and Devasagayam TP: Antioxidant and prooxidant nature of hydroxycinnamic acid derivatives ferulic and caffeic acids. Food Chem Toxicol 48: 3369-3373, 2010.

3. Marques MPM, Borges F, Sousa J, Calheiros R, Garrido J, Gaspar A, Diniz C and Fresco P: Cytotoxic and COX-2 inhibition properties of hydroxycinnamic derivatives. Lett Drug Des Dis 3: 316-320, 2006

4. Mazzone C, Russo N and Toscano M: Antioxidant properties comparative study of natural hydroxycinnamic acids and structurally modified derivatives: Computational insights. Comput Theor Chem 1077: 39-47, 2016.

5. Tavares-da-Silva EJ, Varela CL, Pires AS, Encarnação JC, Abrantes AM, Botelho MF, Carvalho RA, Proença C, Freitas M, Fernandes E and Roleira FM: Combined dual effect of modulation of human neutrophils' oxidative burst and inhibition of colon cancer cells proliferation by hydroxycinnamic acid derivatives. Bioorg Med Chem 24: 3556-3564, 2016.

6. Esteves M, Siquet C, Gaspar A, Rio V, Sousa JB, Reis S, Marques MP and Borges F: Antioxidant versus cytotoxic properties of hydroxycinnamic acid derivatives-a new paradigm in phenolic research. Arch Pharm (Weinheim) 341: 164-173, 2008.

7. Bailey D, Kirby BP, Atkinson J, Fixon-Owoo S, Henman MC, Shaw GG and Doyle KM: Hydroxycinnamic acid amide derivatives of polyamines reverse spermine-induced CNS excitation. Pharmacol Biochem Behav 133: 57-64, 2015.

8. Yeggoni DP, Gokara M, Manidhar DM, Rachamallu A, Nakka S, Reddy CS and Subramanyam R: Binding and molecular dynamics studies of 7-hydroxycoumarin derivatives with human serum albumin and its pharmacological importance. Mol Pharm 11: 1117-1131, 2014.

9. Urtti A: Challenges and obstacles of ocular pharmacokinetics and drug delivery. Adv Drug Deliv Rev 58: 1131-1135, 2006.

10. Meng FY, Zhu JM, Zha AR, Yu SR and Lin CW: Synthesis of p-hydroxycinnamic acid derivatives and investigation of fluorescence binding with bovine serum albumin. J Lumin 132: 1290-1298, 2012.

11. Qi ZD, Zhang Y, Liao FL, Ou-Yang YW, Liu Y and Yang X: Probing the binding of morin to human serum albumin by optical spectroscopy. J Pharm Biomed Anal 46: 699-706, 2008.
12. He W, Li Y, Xue C, Hu Z, Chen X and Sheng F: Effect of Chinese medicine alpinetin on the structure of human serum albumin. Bioorg Med Chem 13: 1837-1845, 2005.

13. Sheldrick GM: SHELXS97 and SHELXL97. program for crystal structure solution and refinement. University of Göttingen, Göttingen. Germany, 1997.

14. He H, Li WD, Yang LY, Fu L, Zhu XJ, Wong WK, Jiang FL and Liu Y: A novel bifunctional mitochondria-targeted anticancer agent with high selectivity for cancer cells. Sci Rep 5: 13543, 2015.

15. He H, Xu J, Cheng DY, Fu L, Ge YS, Jiang FL and Liu Y: Identification of binding modes for amino naphthalene 2-cyanoacrylate (ANCA) probes to amyloid fibrils from molecular dynamics simulations. J Phys Chem B 121: 1211-1221, 2017.

16. Petitpas I, Bhattacharya AA, Twine S, East M and Curry S: Crystal structure analysis of warfarin binding to human serum albumin: Anatomy of drug site I. J Mol Biol 276: 22804-22809, 2001.

17. Humphrey W, Dalke A and Schulten K: VMD: Visual molecular dynamics. J Mol Graph 14: 33-38, 1996.

18. He H, Xu J, Xie W, Guo QL, Jiang FL and Liu Y: Reduced state transition barrier of CDK6 from open to closed state induced by Thr177 phosphorylation and its implication in binding modes of inhibitors. Biochim Biophys Acta 1862: 501-512, 2018.

19. Tong J, Tian F, Liu Y and Jiang F: Comprehensive study of the adsorption of an acylhydrazone derivative by serum albumin: unclassical static quenching. RSC Adv 4: 59686-59696, 2014.

20. Cao H, Jia X, Shi J, Xiao J and Chen X. Non-covalent interaction between dietary stilbenoids and human serum albumin: Structure-affinity relationship, and its influence on the stability, free radical scavenging activity and cell uptake of stilbenoids. Food Chem 202: 383-388, 2016.

21. Hu YJ, Liu Y, Pi ZB and Qu SS: Specific activation of mGlu2 induced IGF-1R transactivation in vitro through FAK phosphorylation. Bioorg Med Chem 13: 6609-6614, 2005.

22. Bhogale A, Patel N, Sarpotdar P, Mariam J, Dongre PM, Miotello A and Kothari DC: Systematic investigation on the interaction of bovine serum albumin with $\mathrm{ZnO}$ nanoparticles using fluorescence spectroscopy. Colloids Surf B Biointerfaces 102: 257-264, 2013.

23. Nishijima M, Pace TC, Nakamura A, Mori T, Wada T, Bohne C and Inoue Y: Supramolecular photochirogenesis with biomolecules. Mechanistic studies on the enantiodifferentiation for the photocyclodimerization of 2-anthracenecarboxylate mediated by bovine serum albumin. J Org Chem 72: 2707-2715, 2017.

24. Anand U, Jash C, Boddepalli RK, Shrivastava A and Mukherjee S: Exploring the mechanism of fluorescence quenching in proteins induced by tetracycline. J Phys Chem B 115: 6312-6320, 2011.

25. Mi R, Hu YJ, Fan XY, Ouyang Y and Bai AM: Exploring the site-selective binding of jatrorrhizine to human serum albumin: Spectroscopic and molecular modeling approaches. Spectrochim Acta A Mol Biomol Spectrosc 117: 163-169, 2014.

26. Gao H, Lei L, Liu J, Kong Q, Chen X and Hu Z: The study on the interaction between human serum albumin and a new reagent with antitumour activity by spectrophotometric methods. J Photochem Photobiol A Chem 167: 213-221, 2004.

27. Ross PD and Subramanian S: Thermodynamics of protein association reactions: Forces contributing to stability. Biochemistry 20: 3096-3102, 1981.

28. Yue Y, Liu R, Liu J, Dong Q and Fan J: Experimental and theoretical investigation on the interaction between cyclovirobuxine $\mathrm{D}$ and human serum albumin. Spectrochim Acta A Mol Biomol Spectrosc 128: 552-558, 2014.

29. Wang Q, Xiao Y, Huang Y and Li H: An important prerequisite for efficient Förster resonance energy transfer (FRET) from human serum albumin to alkyl gallate. RSC Adv 6: 36146-36151, 2016.

30. Fan XY, Zhang Y, Wang J, Yang LY, Jiang FL and Liu Y: Exploring the interaction between rotenone and human serum albumin. J Chem Thermodyn 69: 186-192, 2014.

31. Pescitelli G, Di Bari L and Berova N: Application of electronic circular dichroism in the study of supramolecular systems. Chem Soc Rev 43: 5211-5233, 2014.

32. Zhang HX and Liu E: Spectroscopic and molecular modeling investigation on the binding of a synthesized steroidal amide to protein. J Lumin 153: 182-187, 2014.

33. Hou H, Qu X, Li Y, Kong Y, Jia B, Yao X and Jiang B: Binding of citreoviridin to human serum albumin: multispectroscopic and molecular docking. Biomed Res Int 2015: 162391, 2015.

This work is licensed under a Creative Commons Attribution-NonCommercial-NoDerivatives 4.0 International (CC BY-NC-ND 4.0) License. 